\title{
Localization System for Large Indoor Environments Using Invisible Markers
}

\author{
Yusuke Nakazato*
}

\author{
Masayuki Kanbara ${ }^{\dagger}$ \\ Nara Institute of Science and Technology
}

Naokazu Yokoya $a^{\ddagger}$

\begin{abstract}
We propose a user localization system that uses invisible markers for wearable augmented reality (AR) in large indoor environments. Wearable AR systems have received a great deal of attention as a new method for displaying location-based information in the real world. For using wearable AR systems, it is necessary to measure the position and orientation of a user using a positioning infrastructure without the undesirable visual effects that arise from merging real and virtual worlds. In addition, the infrastructure of the localization environment must be constructed easily and cheaply. The proposed system can estimate the position and orientation of a user precisely by affixing wallpapers containing printed invisible markers on ceilings or walls. The user's position and orientation are estimated by recognizing the markers using an infrared camera with infrared LEDs. To construct environments for the localization system, we developed an initialization tool that calibrates the alignment of the markers from photographs taken by flash illumination using a digital still camera.
\end{abstract}

CR Categories: H.5.1 [Information Interfaces and Presentation]: Multimedia Information Systems - Artificial, augmented, and virtual realities; I.5.4 [Pattern Recognition]: Applications-Computer vision

Keywords: localization, invisible marker, augmented reality

\section{Introduction}

One of the localization methods uses images captured by the user's view camera and a feature database of a real environment. These methods estimate the user's position and orientation by matching features on an image from the user's viewpoint and the known feature points or a 3D model of the environment [Klein and Murray 2007]. However, it is difficult to estimate the user's position in environments that have many similar patterns.

Another approach estimates the user's position and orientation by recognizing visual markers affixed on the ceilings or walls [Naimark and Foxlin 2002]. In this method, it is relatively cheap to set up visual markers to form a positioning infrastructure. In addition, it is possible to estimate the user's position and orientation robustly, because recognition of the markers from an image captured using a wearable camera is relatively easy. However, a visual marker is often so prominent that it creates undesirable visual effects. Therefore, invisible markers[Park and Park 2004; Nakazato et al. 2006] or markers designed to blend with the scene[Saito et al. 2007; Tenmoku et al. 2007] need to be used. These methods can achieve localization without disturbing the scenery.

In order to realize a localization system in indoor environments in practice, we propose a localization system and marker initialization tool using invisible markers. The proposed method has two phases as shown in Figure 1: The first phase is the initialization of invisible-marker environments, and the second phase is installing a user localization system in these environments.

\footnotetext{
*e-mail:nakazato.yusuke@canon.co.jp (Presently with Canon Inc.)

†e-mail:kanbara@is.naist.jp

†e-mail:yokoya@is.naist.jp
}

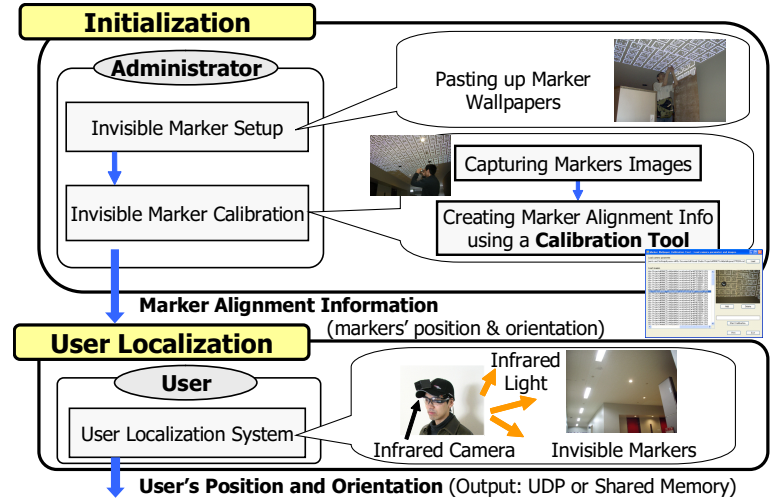

Figure 1: Overview of a user localization system using invisible markers.

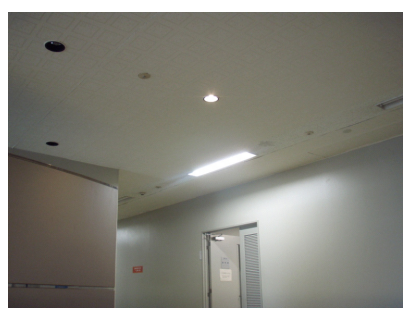

(a) Capturing without a flash.

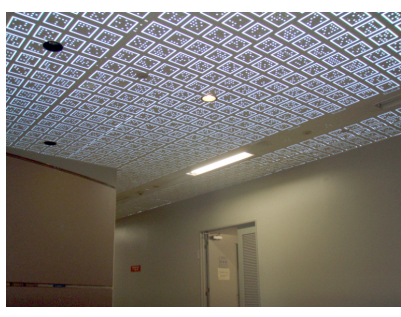

(b) Capturing with a flash.
Figure 2: Environment where Invisible markers wallpaper is installed on the ceiling.

\section{Localization System Using Invisible Mark- ers}

\subsection{First phase: Initialization}

\subsubsection{Invisible Marker Setup}

In this system, wallpapers with pre-printed invisible markers are used because it is easy to set them up. Figure 2 shows an example of an environment where an invisible-marker wallpaper is installed on a ceiling. In general, the user cannot see the markers, as shown in Figure 2(a). However, the markers on the wallpaper can be captured clearly using a camera with a flash because the markers consist of retro-reflectors.

The markers are square, and their inside contains evenly spaced $N \times N$ grid points, and dots are allocated to the grids. To determine the direction of the marker uniquely, one dot is allocated to one of the four corners of the grids, and the other three corners are blank. These dots correspond to bits, which are encoded using a cyclic redundancy check (CRC) error-detecting code. If the number of CRC check bits is $\mathrm{C}$, the total number of IDs associated with the IDs is $2^{N^{2}-4-C}$.

It is difficult to print markers that have unique ID patterns because of the marker printing process. Therefore, all markers on the wallpaper have a pattern in which all bits are initially 1 . The administrator creates a unique ID pattern using the proposed pattern-creating tool. The tool outputs marker design information, which includes a table of random ID patterns. The administrator scratches dots that 


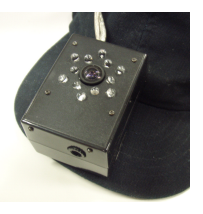

(a) Infrared camera L.

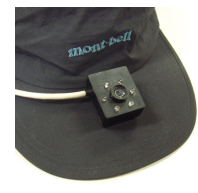

(b) Infrared camera $S$.
Figure 3: Infrared camera with infrared LEDs.

Table 1: Specifications of the infrared camera.

\begin{tabular}{c||c|c}
\hline \hline & IR camera L & IR camera S \\
\hline \hline size[mm] & $75 \times 55 \times 47$ & $25 \times 20 \times 10$ \\
\hline weight[g] & 115 & 20 \\
\hline resolution[pixel] & $1024 \times 768$ & $640 \times 480$ \\
\hline view angle ${ }^{\circ}$ ] & 110 & 70 \\
\hline number of LEDs & 12 & 6 \\
\hline interface & USB2.0 $(\times 2)$ & USB2.0 \\
\hline frame rate[fps] & 29.12 & 30 \\
\hline \hline
\end{tabular}

correspond to 0 bits on the marker, and then the wallpaper is affixed onto the ceilings or walls.

\subsubsection{Invisible Marker Calibration}

In the marker-based approach, the alignment information of markers, i.e., the position and orientation of markers located in the real environment, is needed in advance. In order to obtain the alignment information easily, the proposed system uses a marker calibration tool. The administrator captures images of invisible markers via a normal digital still camera, and the images are inputted into the calibration tool in order to estimate the position and orientation of the markers. The administrator also inputs intrinsic camera parameters, the position and orientation of reference markers, and the markers' design information, which consists of the size, interval of the markers, grids, number of CRC checkbits, and junction information of markers' ID patterns. Finally, the tool outputs marker alignment information, i.e., the markers' position and orientation in the real world. This information is used in the localization phase to estimate the user's position and orientation.

\subsection{Second phase: User Localization}

In the proposed system, the user's position and orientation can be estimated from the intrinsic parameters of an infrared (IR) camera and the marker alignment information from the initialization phase. The user is equipped with a wearable computer, and an IR camera with IR LEDs is fitted on his head. The IR camera captures invisible markers clearly by detecting the reflectance of IR light. The estimated position and orientation of the user can then be written to shared memory on the wearable computer, or can be sent to another computer using UDP connection.

We developed two types of infrared cameras, as shown in Figure 3 . The specifications of these cameras are shown in Table 1 . One camera (IR camera L) has a high resolution and wide angle in order to estimate the position and orientation of the user precisely. The camera also has user's view camera for AR in front of the camera. The other camera (IR camera S) is small and lightweight.

\section{Experiment of User Localization}

We evaluated the accuracy of the estimated position and orientation of markers using the calibration tool. In this experiment, invisiblemarker wallpaper with an area of about $65 \mathrm{~m}^{2}$ was affixed on the ceiling, as shown in Figure 2. The markers with an area of $16 \mathrm{~cm}$ and unique IDs were aligned on the wallpaper. The density of markers was about 24 markers $/ \mathrm{m}^{2}$. The number of marker grids was 5 , and the number of CRC check bits was 3. One marker's position and orientation was input as the reference marker, and 100
Table 2: Errors in estimated position and orientation of the camera under the invisible marker wallpapers.

\begin{tabular}{c|c||c|c}
\hline \hline \multicolumn{2}{|c|}{} & IR camera L & IR camera S \\
\hline \hline \multirow{2}{*}{ position } & average error & $9 \mathrm{~mm}$ & $13 \mathrm{~mm}$ \\
\cline { 2 - 4 } & standard deviation & $4 \mathrm{~mm}$ & $6 \mathrm{~mm}$ \\
\hline \multirow{2}{*}{ orientation } & average error & $0.41^{\circ}$ & $0.52^{\circ}$ \\
\cline { 2 - 4 } & standard deviation & $0.13^{\circ}$ & $0.31^{\circ}$ \\
\hline \hline
\end{tabular}

images $(3072 \times 2304$ pixel $)$ of markers captured by a digital still camera (Olympus, $\mu 770 \mathrm{SW}$ ) were input. The intrinsic camera parameters were estimated via Tsai's method in advance. The grand truths of the markers' position and orientation were manually measured using a total station (Topcon, GPT-9005A, accuracy: $\pm 5 \mathrm{~mm}$ ). In this experiment, the error in the estimated marker positions of marker alignment information was about $13 \mathrm{~mm}$, and standard deviation was about $12 \mathrm{~mm}$.

In the next experiment, we evaluated the accuracy of the estimated position and orientation of the two types cameras in an environment that was constructed in the initialization phase. These cameras were directed toward the ceiling (the distance between the cameras and the ceiling was about $1.2 \mathrm{~m}$ ), and its position and orientation were estimated using the alignment information, which was outputted through the initialization experiment. The true positions and orientations were measured manually using the total station. Table 2 shows the error and standard deviation of the estimated position and orientation of the cameras. In this experiment, the frame rate of localization was about $29 \mathrm{fps}$ (CPU utilization: 15\%) when we use a ThinkPad X60 (Lenovo; CPU: Intel T7200 2 GHz, memory: 2 GB) as a wearable computer.

\section{Conclusion}

We proposed a user localization system and initialization tool using invisible markers for wearable AR. Since it is possible to affix numerous markers closely using an invisible-marker wallpaper, this system can estimate the position and orientation of a user precisely. Furthermore, this system assists the administrator in calibrating the markers using the initialization tool. In experiments, we have confirmed that the proposed system is able to estimate the position and orientation of a user with high accuracy.

\section{References}

Klein, G., AND MurRAY, D. 2007. Parallel tracking and mapping for small AR workspaces. Proc. 6th IEEE/ACM Int. Symp. on Mixed and Augmented Reality, 225-234.

NAIMARK, L., AND FoXlin, E. 2002. Circular data matrix fiducial system and robust image processing for a wearable visioninertial self-tracker. Proc. 1st IEEE/ACM Int. Symp. on Mixed and Augmented Reality, 27-36.

NAKAZATO, Y., KAnBARA, M., AND YoKoya, N. 2006. An initialization tool for installing visual markers in wearable augmented reality. Proc. 16th Int. Conf. on Artificial Reality and Telexistence, 228-238.

PARK, H., AND PARK, J. 2004. Invisible marker tracking for AR. Proc. 3rd IEEE/ACM Int. Symp. on Mixed and Augmented Reality, 272-273.

Saito, S., Hiyama, A., TANiKawa, T., And Hirose, M. 2007. Indoor marker-based localization using coded seamless pattern for interior decoration. Proc. IEEE Virtual Reality 2007, 67-74.

Tenmoku, R., Yoshida, Y., Shibata, F., Kimura, A., And TAMURA, H. 2007. Visually elegant and robust semi-fiducials for geometric registration in mixed reality. Proc. 6th IEEE/ACM Int. Symp. on Mixed and Augmented Reality, 261-262. 